\title{
The accelerated use of online learning during the COVID-19 pandemic
}

\author{
D.H. Tjahjono*, K. Anggadiredja, M. Singgih \\ School of Pharmacy, Bandung Institute of Technology, Indonesia
}

\author{
Keywords \\ Teaching Methods \\ Online Learning \\ Forced Situation \\ Indonesia
}

${ }^{*}$ Corresponding author:

daryonohadi@fa.itb.ac.id
Summary: Most higher education institutions were not ready for online learning in the early days of the COVID-19 pandemic. The School of Pharmacy Bandung Institute of Technology usually ran online learning for no more than $5 \%$ of the total courses (subjects) offered to students. The pandemic has forced learning activities to be delivered online for almost all subjects. For the next semester (autumn 2020) facilities and teaching materials for online learning need to be improved to satisfy stake holders, and to reach equal learning outcomes for students.

\section{Background and Context}

The COVID-19 pandemic is first and foremost a health crisis. It has also affected educational systems worldwide, leading to the near-total closure of schools, universities and colleges. Most governments around the world have temporarily closed educational institutions in an attempt to prevent the spread of COVID-19 (UNESCO, 2020). Teaching has moved online, on an untested and unprecedented scale. Student assessments have also moved online, with a lot of trial and error and uncertainty for everyone.

The same conditions have also occurred in Indonesia; all universities have closed, and the learning process is being conducted online, combining both synchronous and asynchronous learning. In this case study the author will share their experiences in running an online learning process at School of Pharmacy, Bandung Institute of Technology, in the early days of the pandemic, and how to improve online material for the next term.

\section{Educational Description}

In the second week of March 2020 a circular from the Ministry of Education and Culture was issued by a recommendation from the Association of Indonesian Pharmacy Higher Education directing that all learning processes be carried out online. Based on those circulars, the School of Pharmacy, Bandung Institute of Technology setup a Standard Operating Procedure (SOP) in line with the University's policies regarding learning activities during the COVID-19 pandemic. The SOP arranged activities as follow:

1. Manage and schedule faculty members and supporting staff to work in the office with strict health protocols (only lecturers and staff who are healthy, have no underlaying disease, and are not more than 55 years old).

2. All classes/lectures are moved to be delivered online (lecturers are free to use synchronous or asynchronous mediums).

3. All laboratory exercises or experimental learning to be postponed, and experimental tutorial to be conducted online.

4. Student assessments including seminar and comprehensive oral exams to be conducted online.

5. Contribute to public education regarding COVID-19 pandemic. 


\section{Outcomes and Recommendations}

The global lockdown of education institutions is going to be the cause of major (and likely unequal) interruption in students' learning as well as disruptions to student assessments. The COVID-19 pandemic has created complex and unique circumstances for education.

University closures have added to the time that most students already spend at home without explicit face-to-face instruction from lecturers. Meanwhile, faculty members were scrambling to adapt content for an online platform. In many ways, the current situation is unprecedented for most people alive today. Most students and faculty members were not ready to adjust to the conditions of the pandemic.

Under normal circumstances, the University runs no more than $5 \%$ of learning activities by online. Thus, faculty members faced a large problem because most lecturers were not ready for online learning. In unexpected situations, lecturers had previously used some online platforms, such as Zoom, Google Meet or Microsoft Teams. In addition, lecturers were not familiar with the University's learning management system (LMS).

As the pandemic is still unfurling and its end is unknown, in light of the School of Pharmacy's experience for one semester (March to June 2020), a number of measures are prepared for learning activities for the coming semester which include:

1. Preparing teaching materials through workshops in line with the University's policy on improving learning processes to meet the needs of students;

2. Experimental learning and its assessment should be designed well;

3. Students' laboratory work should be facilitated with priority given to doctoral students; and if conditions improve, master's and undergraduate students will then be given a chance to carry out activities on campus although it must be scheduled carefully for their safety.

In addition, by increasing the percentage of online learning, without reducing student learning outcomes, it will provide an opportunity for faculty members to do more research activities and community services as well as to create new collaborations with new partners.

\section{References}

UNESCO (2020). COVID-19 Educational Disruption and Response. Available at: https://en.unesco.org/news/covid-19-educationaldisruption-and-response 